(C) 2020 IEEE. Personal use of this material is permitted. Permission from IEEE must be obtained for all other uses, in any current or future media, including reprinting/republishing this material for advertising or promotional purposes, creating new collective works, for resale or redistribution to servers or lists, or reuse of any copyrighted component of this work in other works. 


\section{Heuristic Evaluation for Virtual Reality for Paediatric Cancer Patient: Perceptions of Healthcare Professionals.}

\author{
$1^{\text {st }}$ Author 1 \\ dept. name of organization (of Aff.) \\ name of organization (of Aff.) \\ City, Country \\ email address or ORCID
}

\author{
$4^{\text {th }}$ Author 4 \\ dept. name of organization (of Aff.) \\ name of organization (of Aff.) \\ City, Country \\ email address or ORCID
}

\author{
$2^{\text {nd }}$ Author 2 \\ dept. name of organization (of Aff.) \\ name of organization (of Aff.) \\ City, Country \\ email address or ORCID
}

\author{
$5^{\text {th }}$ Given Name Surname \\ dept. name of organization (of Aff.) \\ name of organization (of Aff.) \\ City, Country \\ email address or ORCID
}

\author{
$3^{\text {rd }}$ Author 3 \\ dept. name of organization (of Aff.) \\ name of organization (of Aff.) \\ City, Country \\ email address or ORCID
}

\begin{abstract}
There has been an increased interest in Virtual Reality (VR) as a medium of distraction for paediatric cancer patients. Paediatric cancer patients that undergo chemotherapy treatments often face episodes of anxiety, depression and isolation due to the lengthy procedure. The use of video games as a medium of distraction has shown positive results as a tool to provide emotional support for paediatric cancer patients during chemotherapy. However, few studies have qualitatively explored the experience of healthcare professionals on VR usability before testing with real patients. This paper reports an experimental evaluation of healthcare professionals' perspective on the use of VR technology for paediatric cancer patients among healthcare professionals. The objectives are to explore the potential impact of VR as a distraction in paediatric cancer treatment procedure and to identify user requirements for VR technology in the related hospital setting. This paper presents the results of focus-group interviews on this topic with healthcare professionals working in paediatric oncology unit. Interview data was analysed using thematic analysis and a heuristic evaluation technique to identify relevant usability design considerations.
\end{abstract}

Index Terms-Virtual Reality, Heuristic Evaluation, Cancer, Serious Games, Distraction, Paediatric

\section{INTRODUCTION}

Virtual Reality (VR) technology has received an increasing interest in many industries because of its immersive capabilities. The interest in VR is driven by its characteristics of making users active participants in a virtual environment created through an all-encompassing head mounted display and motion tracked controls. VR gives the player the opportunity to explore situations from new perspectives and connect with people from all over the world using the same shared, fullbody, virtual space. Multiple VR applications cater to different sectors, such as education [1] and healthcare [2] [3], in the form of serious games.
In the health industry, VR has been used for pain management and treatment of psychological conditions, such as anxiety and phobias [4]. Additionally, VR has been used to increase wellbeing among elderly at risk of falling [5]. These applications have demonstrated the benefit of immersive VR technology in improving health-related outcomes and maintaining wellbeing of patients. However, there is very little research in using VR for paediatric cancer patients as a medium of distraction while they undergo chemotherapy. Research on the importance of VR as a tool to assist paediatric cancer patients is still scarce and further research is needed to fully understand the use of VR technology with these patients, both at home and in the hospital.

This study hence focuses on gathering data from health professional to assess the feasibility of using VR with paediatric patients throughout chemotherapy procedures. For this preliminary study, health professionals were chosen as they are in direct contact with patients during the treatment and can give an external, expert opinion on the experience of many patients. The implementation of a heuristic evaluation based upon interviews with the health professionals will provide insight towards the feasibility of this practice.

Herein we report the findings of the heuristic evaluation of VR technology from the perspective of 6 health professionals including medical officers, head nurses and nurses. We conducted a series of recorded and transcribed semi-structured interviews where the suitability of the user experience of a specific headset and a game were assessed. The aim of this work is to create initial usability guidelines for designing VR experiences for this context, with future studies aiming to build upon these guidelines through direct interactions with paediatric cancer patients.

This paper provides an overview of previous studies related 
to this topic, the methodology used, the heuristic evaluation and the key findings from our interviews. The rest of the paper is structured as follows. Section Background provides an overview of previous studies related to this topic. In the Methodology section, we describe our approach on heuristic evaluation in details. Next the results of the study are presented and discussed, followed by our conclusion.

\section{BACKGROUND}

Immersive technology such as VR has received an increasing research interest in recent years [2] as it has been utilized to solve various issues and tasks across the healthcare industry to enhance engagement and adherence to treatment. Here, VR has been used for different purposes: as a teaching medium [6], for handling pain of patients [6],and for healing of psychological disorders [7] among others. VR has proven to be an effective tool in assisting with psychology treatment such as Schizophrenia [8] and to overcome anxiety disorders [9].

Research on the efficacy of VR-based intervention as a medium of distraction while the paediatric cancer patients undergo chemotherapy is scarce. In a meta-analysis examining the efficacy of VR-based interventions in cancer-related symptom management, it was reported that VR-based interventions had statistically significant effects on reducing symptoms of anxiety, depression, pain, and statistically significant benefit was observed for fatigue and cognitive function [10]. However, many of the studies that were included had gaps in their methodologies and, therefore, further research is needed to clearly understand the usability considerations and potential benefits of VR technology for these children, especially during hospital admissions.

Prior to evaluating the feasibility of immersive VR as a distraction intervention in a cohort of paediatric cancer patients undergoing chemotherapy procedures, we evaluated this concept with a group of health professionals that have direct contact with patients during treatment in the ward. In this preliminary study, we used the heuristic evaluation technique to assess the feasibility of VR in this application domain. This is a well-defined yet informal way of evaluating the usability of User Interfaces (UIs). Nielsen and Molich [11] describe heuristic evaluation as the process of the practitioner looking at the UI and judging its quality based on their own knowledge and experience. Thus, it is an inspection technique where a set of usability principles is established and used by evaluators to explore an interface, where these principles are called heuristic [12].

\section{Methodology: Heuristic Evaluation}

The heuristic evaluation [13] is divided into several steps. These are: (1) the identification of usability issues in VR and their categorization; (2) the observation of players while interacting with VR under the observation of an evaluator; (3) interview and recording the answers; (4) re-categorization of usability based on the answers; and (5) creation of new heuristics. These stages are visualised in Figure 1.

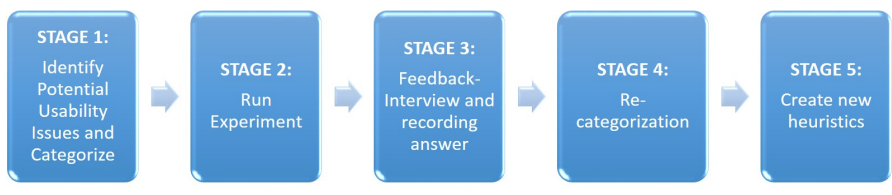

Fig. 1. Stages of Heuristic Evaluation

This study used the heuristic evaluation technique to determine the effectiveness and usability of a VR game as a medium of distraction for paediatric cancer patients undergoing chemotherapy. The main aim of this evaluation is to investigate health professionals' experiences and perceptions of VR as linked to paediatric cancer patients' experiences. Heuristic evaluation of VR games was conducted through several stages in this study based on Figure 1.

\section{A. Stage 1: Category Definition}

Our categories were based upon the Multi-criteria Assessment of Usability for Virtual Environments (MAUVE) [13]. MAUVE is a taxonomy of virtual environment heuristics that aims to produce an organized way of attaining effective usability and engaging user experiences when creating VR experiences. Criteria in the MAUVE system include: wayfinding, navigation, object selection and manipulation, visual output, auditory output, haptic output, simulator-sickness, engagement, presence, and immersion.

We developed a bespoke subset list of criteria from MAUVE which contains a general categorization that are suitable to apply for VR games in our given context. We developed interview questions based upon this categorization, which included a) Object Selection and Manipulation, b) Visual and Audio Output, c) Comfort, d) Engagement, and e) Immersion.

a) Object Selection and Manipulation: The ability to select and manipulate objects inside a virtual environment. Similarly to traditional graphical user interfaces, comprehending, selecting and optimizing the ways in which users utilize and interact with items in VR environments and solve respective challenges, is a crucial part of creating successful virtual experiences [14].

b) Visual and Audio Output: Users can rely on their sense of vision and hearing to observe the cues around them. When it comes to computer interfaces, it has been shown that appropriately organized visual and audio cues can effectively guide and captivate users in VR [15].

c) Comfort: The importance of users feeling comfortable when wearing head-mounted displays, as potential physical pain can create negative thoughts and emotions regarding the experience. Discomfort in virtual reality can be the outcome of both physical (such as the weight of the device) and visual (such as poorly rendered imagery) characteristics of the system, and can affect the usability and overall experience [13]. 
TABLE I

PROFILE OF EXPERT EVALUATORS

\begin{tabular}{|c|c|l|}
\hline No & ID & \multicolumn{1}{c|}{ Professional Role } \\
\hline 1 & HP1 & $\begin{array}{l}\text { Medical Officer- Paediatric specialist trainee in year 1, } \\
\text { doing Oncology rotation. }\end{array}$ \\
\hline 2 & HP2 & $\begin{array}{l}\text { Medical Officer- Paediatric specialist trainee in year 3, } \\
\text { doing Oncology rotation. }\end{array}$ \\
\hline 3 & HP3 & Head of Nurse \\
\hline 4 & HP4 & Head of Nurse \\
\hline 5 & HP5 & Nurse \\
\hline 6 & HP6 & Nurse \\
\hline
\end{tabular}

d) Engagement: The level to which players are engaged with the experience that is presented to them. A higher level of engagement can lead to better performance on required tasks and better retention of information presented by the experience.

e) Immersion: The level to which user inhabit the virtual space and ignore the real world. Immersion can be described as the compilation of technological components of a system that serves the purpose of achieving an engrossing, captivating and evocative representation of reality to the users [16].

\section{B. Stage 2 - Observation of players while interacting with VR under the observation of an evaluator.}

Selected health professional participated in the evaluation. Six health professional were involved including medical officers, head nurses and general nurses. They were selected based on their experience related to paediatric cancer patients at the Paediatric Oncology Ward, Hospital Tuanku Mukhriz (UKM Medical Center), Kuala Lumpur, Malaysia. Table 1 shows the profile of each expert evaluator.

At this stage, the qualitative methodology adopted used a semi-structured interview in a focus group setting. The first part of the interview regarded the procedures in chemotherapy for paediatric cancer patients, as well as the patients' experiences that involved interactive technologies during the procedure. Next, a demonstration of a VR headset was performed by one of our researchers. The VR game named Beat Saber was used in the demonstration.Interface of Beat Saber as shown in Figure 2. Beat Saber is VR rhythm game where the goal is to slash the beats that are represented as small cubes as they are coming towards the player. This game is a well regarded VR game that makes use of large, full body movements while standing in one spot. Because of this mobility, it is noted that this game may not be appropriate for paediatric cancer patients during chemotherapy procedures, but would be a good exergame to get patients physically active during prolonged stays in the ward. After the demonstration, participants were optionally asked whether they wished to experience a five minute VR game using the Oculus Quest Headset and all participant agree to play with Beat Saber VR game. Figure 3 shown health professionals playing VR game. After participants experienced the VR games, they were invited for a follow-up group interview on their experience.

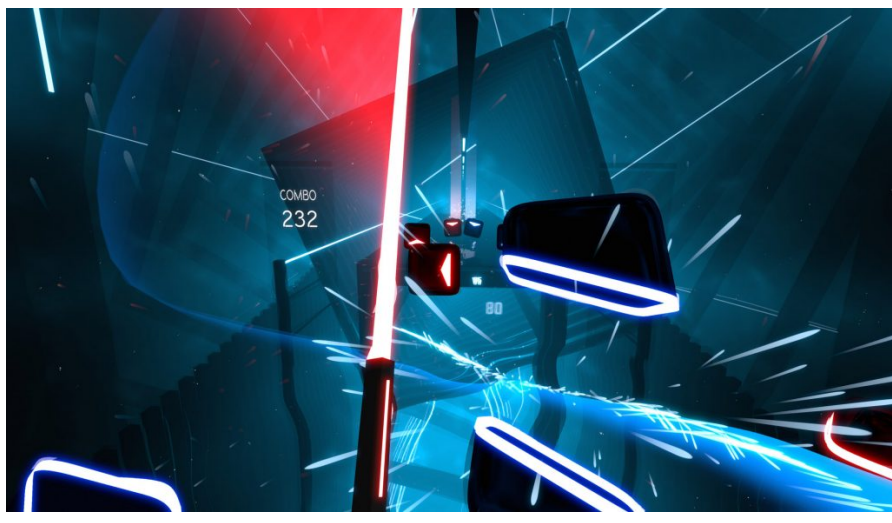

Fig. 2. Beat Saber VR Game interface. Photo from https://www.roadtovr.com/beat-saber-early-access-review-vr-rhythm-gamebudding-jedi-knights/
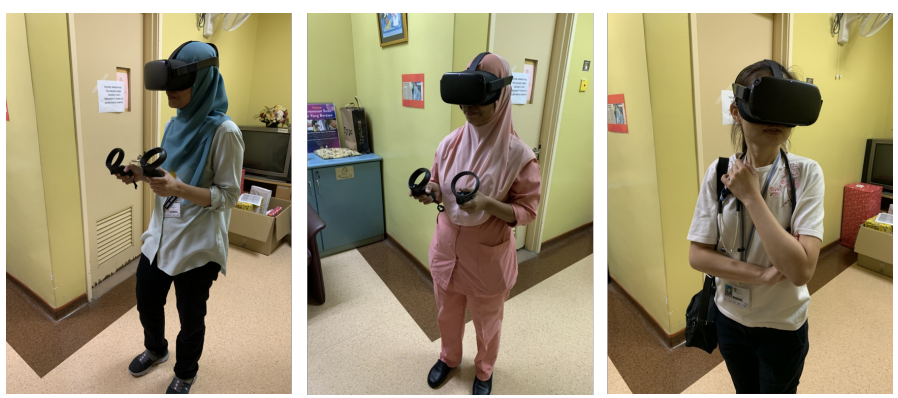

Fig. 3. Health professionals experience play VR game

\section{Stage 3 - Interview and recording the answers}

All the interviewees were asked for their consent to be involved in the study, which was conducted on the 24th Feb 2020. In total, 11 semi-structured interview questions were asked after the VR demonstration and specifically regarding VR technology. The qualitative answers were voice recorded during the interviews and then were transcribed and translated into English. The voice records were then transcribed and underwent thematic analysis, a method for identifying, analysing and reporting meaningful patterns within unstructured data [17].This is to identify the key concepts expressed by the interviewees that will be used to determine heuristics.

\section{Stage 4: Re-categorization of usability based on the an-} swers

All 11 interview questions analysed here were based on VR experience after the participants played the Beat Saber game. The questions were based on how players handled an object and manipulated it, the satisfaction on visual and audio experience, the comfort of the headset, and to see how engaged and immersed the players felt while playing the VR game. We categorized the answers using thematic analysis, with preconstructed themes based upon the subset of MAUVE criteria discussed earlier. After running the evaluation and categorizing the answers, we concluded that the health professionals mentioned the five MAUVE criteria a total of 20 times. Each health professional found around 3 criteria on 
TABLE II

INTERVIEW ANSWERS BASED ON HEURISTIC CRITERIA

\begin{tabular}{|c|c|c|c|c|c|c|c|}
\hline Criteria & \multicolumn{6}{|c|}{ Health Professional (HP) } & Total \\
\hline & HP1 & HP2 & HP3 & HP4 & HP5 & HP6 & \\
\hline $\mathbf{a}$ & & 1 & & & & 1 & 2 \\
\hline $\mathbf{b}$ & 1 & 1 & 1 & 1 & 1 & & 5 \\
\hline c & 1 & 1 & & 1 & 1 & & 4 \\
\hline d & 1 & & 1 & 1 & & 1 & 4 \\
\hline $\mathbf{e}$ & & 1 & 1 & 1 & 1 & 1 & 5 \\
\hline Total & 3 & 4 & 3 & 4 & 3 & 3 & 20 \\
\hline
\end{tabular}

average and the range of list of criteria seen for each health professional was between 1 and 5 based on Table 2. In Stage 5 below, these themes and the linked comments made by the health professionals are used to refine the heuristics for VR usability for paediatric cancer patients in the hospital.

Table 2 shows the frequency of interview answers for each category. It was found that the main criteria that most health professionals are concerned with was related to the visual and audio output experience and the immersion of VR games. In the study, one of expert stated that "the 360 view is amazing and supported by great audio and visual effects. I feel like I in the virtual world. I love this experience and keen to try it again and play a lot of games and maybe watch movie". The VR game used here, Beat Saber, was able to immerse the user in virtual reality experience, specifically through visual fidelity and interactive, surround-sound VR audio.

\section{E. Stage 5: Create new heuristics}

After categorization, we developed our heuristics. The final heuristics are listed below:-

1) Establish relation between visual and audio to create a quality VR experience - The content and objects in VR games should have a combination of visual effects and audio. For example, each object should have a clear audio cue and a voice explanation when player selects the object to give a better understanding the user's interaction with the virtual environment. Similarly, Ahrens et al. [18]stated that presenting visual information of hand-location and room dimensions showed better sound localization performance compared to the condition with no visual information.

2) Establish relation between visual experience and immersion - The game should have the best visual experience possible in order to immerse players in the gameplay. VR systems have been shown to be capable of faithfully reproducing visual environments and providing reliable spatial tracking accuracy of head and hand motion, both of which contribute to a strong sense of immersion [19].

3) Establish appropriate comfort zone and condition of the patients - The patients should not feel uncomfortable with the VR headset device during the treatment. Avoid unnecessary movement that involves physical coordination. Yan et al. [20] stated that weight, headset position, and pressure of the headset are the primary causes of discomfort in VR devices.

4) Training phases - All participants were first time users of VR and they required a training session on how to use the controller and manipulate objects in the VR environments. Bowman et al. [14] highlighted that one of the most crucial characteristics when it comes to interaction in virtual environments is how consistent the interactions that occur in the real world are in relation to their digital counterparts, allowing users to more easily learn how the virtual world operates.

\section{RESULT}

We now map the appropriate proposed heuristic to address each particular MAUVE criterion. The main criteria to consider for usability in VR is the visual output of the game. To observe the world around us, we rely on our sense of vision and hearing to detect cues in our environment. Almost all participants agree that the combination visual effects and audio are important to make them immerse in the game play. One of the experts stated that the experience was "so real, especially $3 D$ graphics and the sound from the headset gives a better feel to play the game". The sound system in Oculus Quest would assist into minimising the awareness of the real world and therefore enable the immersion into the virtual world, as a way of optimising the experience.

The second criteria to consider for usability in virtual reality is the immersion in the game play. Geszten et al. [21] explain that realistic 3D environments can give impact to behaviours of players due to the similarities that can be found between in how we interact with the actual world around us and the possibilities offered in a virtual environment. Most of health professional in this study agreed that patients will immerse in the gameplay and forget what is happening in the real world. One of the participant stated that "kids are crazy with games. Out patients too. I think yes, patients will immerse and focus while playing".

The next criteria that was considered is the comfort of the user when using VR. McCauley-Bell [22] states that ensuring overall pleasantness and well-being when users interact with Virtual Reality equipment is of utmost priority. In this study, one of the experts stated that "the VR set is quite heavy for me and when using it, in certain times I feel a bit of pain in my neck. Patients will love it but I am wondering how they can carry this headset because most of them are still young". Factors that cause discomfort, such as continuous usage and weight of the headset, can impact how the users feel during a usability testing session as well as during normal use. Durlach and Mavor [23] state that users should feel comfortable when wearing a head-mounted display to avoid pain and unpleasantness that can create negative thoughts and emotions. Regarding the above, it was suggested that VR should establish appropriate comfort zone that give patients a feeling of comfort with the device during the treatment (Heuristic 3).

The last criteria to consider for usability in VR is the object selection and manipulation. Similarly to traditional graphical user interfaces, comprehending, selecting and optimizing the ways in which users utilize and interact with items in virtual environments and solve respective challenges, is a crucial part of creating successful virtual experiences. Three out of 
5 health professional agreed that they experienced difficulty when handling with the object selection and manipulation in the games because all of them are first time users of VR. One of the participants stated that "personally is a bit hard for me as a first timer to play a VR game", resulting from the lack of training to use VR set as a first timer user. Consequently, it was found that the user require training allowing the patients to get familiar with the use of VR set and how to handle and use the controller. (Heuristic 4).

\section{DISCUSSION}

As the result in this study, the use of VR technology and games for the purpose of distraction among paediatric cancer patients requires a deep inspection to guarantee optimum results on wellbeing of the patients. The use of heuristic evaluation to assess the usability of VR technology and games, and the insight of health professionals, revealed some solutions and results about how VR intervention can be used for paediatric cancer patients. In VR, head-mounted displays serve the purpose of entirely concealing the visual field of a user to immerse them in a realistically generated virtual environment or video experience [24].

In Virtual Reality, researchers have been exploring and appraising solutions that allow us to interact and manipulate objects in 3D environments [25] [26].

The combination of wearing a VR head set for large amounts of time, small space for mobility within an area and navigating said space can potentially involve a level of physical risk for users. Sharples et al. [27] stated that not paying attention to the usability of the Virtual Reality control devices and how the devices eventually interact with the interface can heavily impact the Virtual Reality application experience.

However, there is still a need for clear design guidelines for high quality VR experiences in diverse usage contexts. Developers and designers must create VR products that are feasible for the context, effective at delivering on their serious game objective, and at the same time the best possible user experience to the players.

\section{CONCLUSION}

The usage of VR technology and games for health purposes has shown positive outcomes. Moreover, as the characteristics that can potentially influence the experience of a user in Virtual Reality can be numerous and overwhelming, User-Experience specialists, developers and researchers working with Virtual Reality need to identify, understand and be aware of the aspects of the technology that can potentially influence the experience of the users and the methods that can evaluate them. This is crucial as the aspects that might be considered important in one application can be irrelevant in another one. As the area of Virtual Reality technology continues to evolve rapidly, efficient yet holistic user-experience evaluations of Virtual Reality can ensure that the solutions provided meet the needs of the users and ensure a full-filling, enjoyable and pain-free experience to the users.

\section{REFERENCES}

[1] H. Maresky, A. Oikonomou, I. Ali, N. Ditkofsky, M. Pakkal, and B. Ballyk, "Virtual reality and cardiac anatomy: Exploring immersive threedimensional cardiac imaging, a pilot study in undergraduate medical anatomy education," Clinical Anatomy, vol. 32, no. 2, pp. 238-243, 2019.

[2] A. Suh and J. Prophet, "The state of immersive technology research: A literature analysis," Computers in Human Behavior, vol. 86, pp. 77-90, 2018.

[3] K. Niki, Y. Okamoto, I. Maeda, I. Mori, R. Ishii, Y. Matsuda, T. Takagi, and E. Uejima, "A novel palliative care approach using virtual reality for improving various symptoms of terminal cancer patients: A preliminary prospective, multicenter study," Journal of palliative medicine, vol. 22 no. 6, pp. 702-707, 2019

[4] T. D. Parsons and A. A. Rizzo, "Affective outcomes of virtual reality exposure therapy for anxiety and specific phobias: A meta-analysis," Journal of behavior therapy and experimental psychiatry, vol. 39, no. 3, pp. 250-261, 2008.

[5] J. A. Garcia, "A virtual reality game-like tool for assessing the risk of falling in the elderly," Stud. Health Technol. Inform., vol. 266, pp. 63-69, 2019.

[6] M. Taubert, L. Webber, T. Hamilton, M. Carr, and M. Harvey, "Virtual reality videos used in undergraduate palliative and oncology medical teaching: results of a pilot study," BMJ supportive \& palliative care, vol. 9, no. 3, pp. 281-285, 2019.

[7] W. H. Wan and A. H. Y. Lam, "The effectiveness of virtual reality-based simulation in health professions education relating to mental illness: A literature review," Health, vol. 11, no. 6, pp. 646-660, 2019.

[8] T. Souto, H. Silva, A. Leite, A. Baptista, C. Queirós, and A. Marques, "Facial emotion recognition: Virtual reality program for facial emotion recognition - a trial program targeted at individuals with schizophrenia," Rehabilitation Counseling Bulletin, vol. 63, no. 2, pp. 79-90, 2020

[9] J. L. Maples-Keller, C. Yasinski, N. Manjin, and B. O. Rothbaum, "Virtual reality-enhanced extinction of phobias and post-traumatic stress," Neurotherapeutics, vol. 14, no. 3, pp. 554-563, 2017.

[10] Y. Zeng, J.-E. Zhang, A. S. Cheng, H. Cheng, and J. S. Wefel, "Metaanalysis of the efficacy of virtual reality-based interventions in cancerrelated symptom management," Integrative cancer therapies, vol. 18, p. $1534735419871108,2019$.

[11] J. Nielsen and R. Molich, "Heuristic evaluation of user interfaces," in Proceedings of the SIGCHI conference on Human factors in computing systems, pp. 249-256, 1990.

[12] J. Nielsen, "How to conduct a heuristic evaluation," Nielsen Norman Group, vol. 1, pp. 1-8, 1995 .

[13] K. M. Stanney, M. Mollaghasemi, L. Reeves, R. Breaux, and D. A. Graeber, "Usability engineering of virtual environments (ves): identifying multiple criteria that drive effective ve system design," International Journal of Human-Computer Studies, vol. 58, no. 4, pp. 447-481, 2003.

[14] D. Bowman, E. Kruijff, J. La Viola, and I. Poupyrev, "I., the art and science of $3 \mathrm{~d}$ interaction, tutorial notes," in Proceedings of IEEE International Virtual Reality 2000 conference. New Brunswick, NJ, 2000.

[15] D. Massiceti, S. L. Hicks, and J. J. van Rheede, "Stereosonic vision: Exploring visual-to-auditory sensory substitution mappings in an immersive virtual reality navigation paradigm," PloS one, vol. 13, no. 7, 2018.

[16] T. Aitamurto, S. Zhou, S. Sakshuwong, J. Saldivar, Y. Sadeghi, and A. Tran, "Sense of presence, attitude change, perspective-taking and usability in first-person split-sphere 360 video," in Proceedings of the 2018 CHI Conference on Human Factors in Computing Systems, pp. 112, 2018.

[17] V. Braun, V. Clarke, N. Hayfield, and G. Terry, "Thematic analysis," Handbook of research methods in health social sciences, pp. 843-860, 2019.

[18] A. Ahrens, K. D. Lund, M. Marschall, and T. Dau, "Sound source localization with varying amount of visual information in virtual reality," PloS one, vol. 14, no. 3, 2019.

[19] A. Borrego, J. Latorre, M. Alcaniz, and R. Llorens, "Comparison of oculus rift and htc vive: feasibility for virtual reality-based exploration, navigation, exergaming, and rehabilitation," Games for health journal, vol. 7 , no. 3, pp. 151-156, 2018

[20] Y. Yan, K. Chen, Y. Xie, Y. Song, and Y. Liu, "The effects of weight on comfort of virtual reality devices," in International Conference on Applied Human Factors and Ergonomics, pp. 239-248, Springer, 2018. 
[21] D. Geszten, B. P. Hámornik, A. Komlodi, K. Hercegfi, B. Szabó, and A. Young, "Qualitative analysis of user experience in a $3 \mathrm{~d}$ virtual environment," Proceedings of the Association for Information Science and Technology, vol. 52, no. 1, pp. 1-4, 2015.

[22] P. McCauley-Bell, "Ergonomics in virtual environments," Handbook of virtual environments: Design, implementation, and applications, pp. 807-826, 2002.

[23] N. Durlach, A. S. Mavor, and G. B. Newby, "Virtual reality: Scientific and technological challenges," Library and Information Science Research, vol. 18, no. 3, pp. 278-280, 1996.

[24] H. Hua, "Enabling focus cues in head-mounted displays," Proceedings of the IEEE, vol. 105, no. 5, pp. 805-824, 2017.

[25] R. W. Soukoreff and I. S. MacKenzie, "Towards a standard for pointing device evaluation, perspectives on 27 years of fitts' law research in hci," International journal of human-computer studies, vol. 61, no. 6, pp. 751789, 2004.

[26] R. J. Teather and W. Stuerzlinger, "Pointing at 3d target projections with one-eyed and stereo cursors," in Proceedings of the SIGCHI Conference on Human Factors in Computing Systems, pp. 159-168, 2013.

[27] S. Sharples, A. W. Stedmon, M. D'Cruz, H. Patel, S. Cobb, T. Yates, R. Saikayasit, and J. R. Wilson, "Human factors of virtual reality-where are we now?," in Meeting diversity in ergonomics, pp. 173-186, Elsevier Science Amsterdam, 2007. 